\title{
Emerging treatments in Castleman disease - a critical appraisal of siltuximab
}

\author{
This article was published in the following Dove Press journal: \\ Biologics: Targets and Therapy \\ 25 January 2016 \\ Number of times this article has been viewed
}

\section{Jean L Koff \\ Sagar Lonial}

Department of Hematology and Medical Oncology, Emory University

School of Medicine, Atlanta, GA, USA
Correspondence: Sagar Lonial Department of Hematology and Medical Oncology, Winship Cancer Institute, Building C, Room 4004, Emory University, 1365 Clifton Road, Atlanta, GA 30322, USA

Tel +l 4047275572

Fax + I 4047785530

Email sloni0I@emory.edu
Abstract: Castleman disease (CD) is a rare, heterogeneous lymphoproliferative disorder for which no standard of care currently exists. Evidence that the pathophysiology of CD is fueled by excessive interleukin-6 (IL-6) has led to considerable interest in therapeutic targeting of this cytokine. Siltuximab, a chimeric monoclonal antibody to IL-6, has thus emerged as a promising treatment option in a disease lacking efficacious therapy. Here, we review the findings of recent studies evaluating single-agent siltuximab treatment in $\mathrm{CD}$, including the first-ever randomized clinical trial in this disease. Although much more work is needed to establish a standardized treatment approach, siltuximab appears to be a safe and effective treatment for patients with newly diagnosed and previously treated CD.

Keywords: treatment, lymphoma, interleukin-6

\section{Introduction}

Castleman disease (CD) comprises a group of rare disorders characterized by proliferation of morphologically benign lymph nodes due to proinflammatory effects of excessive cytokines, especially interleukin-6 (IL-6). Due to the rarity and heterogeneity of the disease, it has been difficult to establish the incidence of $\mathrm{CD}$; however, a recent study estimates that 4,253 new cases of CD are diagnosed in the US each year. ${ }^{1}$

As shown in Figure 1, CD is broadly divided by clinical presentation into unicentric disease, involving only a single lymph node, and multicentric disease, which causes generalized lymphadenopathy with systemic symptoms including fatigue, cachexia, and fever, as well as laboratory abnormalities, including anemia and increased concentrations of acute-phase proteins (eg, C-reactive protein [CRP] and fibrinogen). ${ }^{2}$ $\mathrm{CD}$ is also categorized in terms of lymph node histopathology, with hyaline-vascular, plasmacytic, and mixed cellularity variants; a fourth variant, plasmablastic, is found only in multicentric CD. The multicentric variant is further distinguished by its association with HIV and human herpesvirus-8 (HHV-8) positivity in many cases. ${ }^{3}$ In fact, recent work has focused on the subclassification of multicentric CD based on association with HHV-8 and/or HIV, a vital distinction, especially as it relates to effective therapy for each entity. ${ }^{4}$

While unicentric CD is largely curable by resection of the affected lymph node, identifying effective therapy for HIV-negative multicentric disease has proved more difficult. Due to the rarity of the disease, randomized clinical trials remain a challenge, and there is no established standard of care for treatment. Treatment strategies have included suppression of immune and inflammatory responses as well as targeted elimination of those cells responsible for producing 


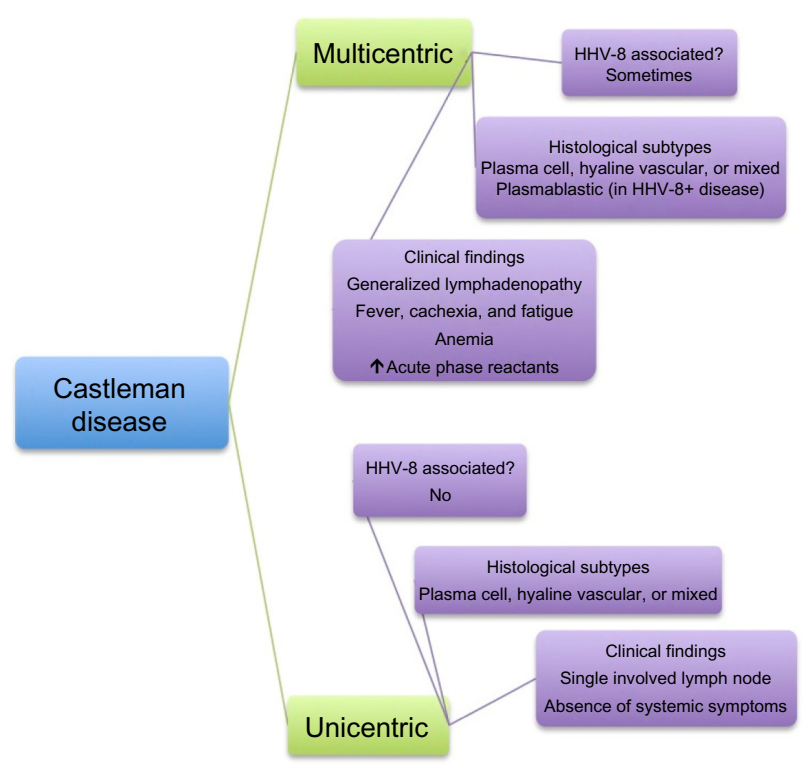

Figure I Historical characterization of Castleman disease. Abbreviation: HHV-8, human herpesvirus-8.

the culprit cytokines. ${ }^{4}$ Unfortunately, while corticosteroids are sometimes effective in subduing a CD flare, patients often relapse upon steroid taper. ${ }^{5,6}$ Immunosuppressive cyclosporine A has also been used, but data on its effectiveness are limited to case reports. ${ }^{7,8}$ Rituximab, a monoclonal CD20 antibody that targets B cells, is well established as effective first-line therapy in HIV-associated $\mathrm{CD},{ }^{9-11}$ but has not been shown to result in sustained responses in HIVnegative disease. ${ }^{12-14}$ Similarly, cytotoxic chemotherapy regimens based on those used in lymphoma (eg, cyclophosphamide, doxorubicin, vincristine, and prednisone, known as CHOP) may induce responses, but these are often short-lived. ${ }^{15,16}$ Although survival data for multicentric CD are not well established, a systematic literature review in 2011 demonstrated a 45.7\% 3-year disease-free survival rate among 84 case reports of HIV-negative multicentric CD. ${ }^{17}$ In a 2012 case series of 60 multicentric CD patients, 10 -year overall survival (OS) was found to be $40 \% .^{18}$

Given the relative failure of conventional therapies in multicentric CD, researchers have looked to IL-6 as a potential therapeutic target. Although the mechanisms underlying dysregulated IL-6 secretion in CD remain unknown, understanding of the pleiotropic effects of IL-6 sheds some light onto the pathophysiology of this poorly understood disease. In addition to its role in hematopoiesis, IL-6 promotes B lymphocyte and plasma cell growth, activates macrophages and T-cells, and mediates hepatic production of inflammatory proteins. IL- 6 also decreases albumin secretion by the liver, which causes a decrease in the oncotic pressure of the blood, leading to pleural or pericardial effusions as well as peripheral edema. ${ }^{19}$ Notably, IL-6 transgenic mice exhibit a CD-like syndrome exemplified by peripheral lymphadenopathy, anemia, hypoalbuminemia, and hypergammaglobulinemia. ${ }^{20}$ Preclinical studies showed that these symptoms could be alleviated by treatment with antibody to IL-6 receptor. ${ }^{21}$

Indeed, over the last decade, drugs that inhibit IL-6 signaling have emerged as a promising new avenue of therapy for multicentric CD. In 2005, Nishimoto et $\mathrm{al}^{22}$ published data demonstrating that tocilizumab, a humanized monoclonal antibody to IL-6 receptor, could produce a durable response in terms of symptoms and laboratory values while maintaining a tolerable safety profile in $C D$ patients; tocilizumab is currently approved for treatment of CD in Japan based on the results of that Phase II, open-label, single-armed study. More recently, the first randomized clinical trial in CD was published in Lancet Oncology, evaluating the effectiveness of the IL-6 antibody siltuximab. ${ }^{23}$ Here, we review the studies investigating siltuximab treatment in $\mathrm{CD}$ and provide a critical appraisal of the data currently available.

\section{Phase I}

The final results of a Phase I trial investigating siltuximab treatment in patients with multiple myeloma, B-cell non-Hodgkin lymphoma, and CD were published by Kurzrock et $\mathrm{al}^{24}$ in Clinical Cancer Research in 2013. The study included 37 patients with either symptomatic multicentric (35) or unresectable unicentric CD (2). None of these patients had HIV-associated disease and only one patient was seropositive for HHV-8.

The study was designed to investigate a total of 7 dose cohorts. Escalating doses of siltuximab infused intravenously over 2 hours $(3 \mathrm{mg} / \mathrm{kg}$ every 2 weeks, $6 \mathrm{mg} / \mathrm{kg}$ weekly or every 2 weeks, or $12 \mathrm{mg} / \mathrm{kg}$ every 2 or 3 weeks) were evaluated in cohorts 1 through 5 . A shorter infusion period of 1 hour, with $12 \mathrm{mg} / \mathrm{kg}$ every 3 weeks was evaluated in cohort 6. Cohort 7 was an extension cohort that included $\mathrm{CD}$ patients only and evaluated siltuximab infused over 1 hour at 12 or $9 \mathrm{mg} / \mathrm{kg}$ every 3 weeks. Response was assessed both radiologically using Cheson criteria ${ }^{25}$ and by clinical benefit response (CBR), which was defined as improvement in at least one of the following criteria: increase in hemoglobin $(\mathrm{Hb}) \geq 2 \mathrm{~g} / \mathrm{dL}$; decrease in fatigue or anorexia by at least one grade; decrease in fever by at least $2^{\circ} \mathrm{C}$, return to normal temperature, or improvement in night sweats; weight increase $\geq 5 \%$; and decrease in diameter of the largest lymph node by $\geq 25 \%$. 
In terms of safety, the authors found no dose-limiting or cumulative toxicities associated with siltuximab infusion. Grade $\geq 3$ adverse events (AEs) attributable to siltuximab included neutropenia $(n=11)$ and thrombocytopenia $(n=3)$; grade $\geq 3$ sepsis and hyperlipidemia occurred in only one patient each. Of note, patients with multiple myeloma were significantly more likely to have grade $\geq 3$ AEs $(69 \%)$ than patients with either non-Hodgkin lymphoma (35\%) or CD (11\%). The most common AEs of all grades included thrombocytopenia (25\%), neutropenia (19\%), hypertriglyceridemia (19\%), leukopenia (18\%), hypercholesterolemia $(15 \%)$, and anemia (10\%). These events led to dose delay or discontinuation in only two cases $(n=1$ each for thrombocytopenia and anemia). Four patients experienced reversible infusion-related reactions, which did not persist or result in discontinuation of treatment. Four patients discontinued drug due to siltuximab-associated AEs, but there were no siltuximab-related deaths.

Interim results in patients with CD were initially reported in the Journal of Clinical Oncology in 2010. ${ }^{26}$ After final analysis, $86 \%$ CD patients improved in $\geq 1$ CBR criterion, with $43 \%$ improving in $\geq 4$ criteria. More specifically, a majority of patients showed improvement in fatigue $(78 \%)$, size of largest lymph node $(65 \%)$, weight (60\%), and fever or night sweats (51\%). Twelve of $36 \mathrm{CD}$ patients had a radiologic response, 1 with complete response (CR) and 11 with partial response (PR). The authors reported that maximum suppression of CRP was reached at the dose of $12 \mathrm{mg} / \mathrm{kg}$ every 3 weeks, and it is notable that 8 of 19 patients treated at that dose achieved response. Interestingly, expression of IL-6 and IL-6 receptor were not found to be related to response. Median OS in all patients was 67.8 months, but was not reached in the CD population.

\section{Open-label Phase II}

Given the promising results of the Phase I trial and the need to evaluate the safety of long-term treatment with siltuximab, the study was extended into an open-label Phase II trial using 19 CD patients with sustained disease control. ${ }^{27}$ Currently, interim results are available only in abstract form. At initiation of the Phase II study, one patient had achieved CR, eleven showed PR, and seven had maintained stable disease. Of these, $68 \%$ had received treatment prior to siltuximab, and 37\% were newly diagnosed. Patients were treated at a dose of $11 \mathrm{mg} / \mathrm{kg}$ every 3 weeks, although dosing interval was increased to every 6 weeks for eight patients who demonstrated prolonged PR or CR.
Median treatment time was 5 years, with $74 \%$ patients treated for 4 years or longer. At a median follow-up time of 5 years, OS was $100 \%$.

Over the entire course of treatment (ie, Phases I and II), the most common AEs were upper respiratory tract infection $(89 \%)$, nausea $(63 \%)$, vomiting (58\%), diarrhea $(53 \%)$, hypercholesterolemia (47\%), hypertriglyceridemia, pain in extremities, headache, rash, and hepatic function abnormalities (each 42\%). Events $\geq$ grade III included gastrointestinal complications (32\%), infections $(26 \%)$, blood/lymphatic system disorders and general disorders/administration-site conditions (each 21\%). Incidence of AEs was higher in the first 2 years of treatment than during years 2-4 or beyond 4 years. Importantly, all patients were alive and maintained disease control at time of reported results.

\section{Randomized, controlled clinical trial}

Results from the very first randomized clinical trial in CD were published in 2014. This multicenter, double-blinded study involved 38 hospitals in 19 countries and enrolled 79 patients with symptomatic multicentric CD; HIVand HHV-8-associated disease was excluded.

\section{Methods}

Patients were randomized 2:1 to receive treatment with siltuximab $11 \mathrm{mg} / \mathrm{kg}$ or placebo every 3 weeks, along with best supportive care. Drug was stopped at treatment failure, which was defined as sustained increase in grade $\geq 2$ disease-related symptoms persisting $\geq 3$ weeks, new disease-related grade $\geq 3$ symptoms, sustained $>1$ point increase in Eastern Cooperative Oncology Group performance status (ECOG-PS) persisting for $\geq 3$ weeks, radiological progression by modified Cheson criteria, or initiation of another treatment for multicentric CD. The primary endpoint for this trial was durable tumor and symptomatic response, including radiologic $\mathrm{CR}$ or PR by Cheson criteria, which were adjusted to account for cutaneous lesions, and improvement or stabilization of CD-related symptoms during $\geq 18$ weeks of treatment. Disease burden was monitored with computed tomography (CT) scan every 9 weeks for the first 6 months, then every 3 months subsequently. Secondary endpoints included duration of response, tumor response, time to treatment failure, $1.5 \mathrm{~g} / \mathrm{dL}$ or greater increase in hemoglobin between baseline and week 13, discontinuation of corticosteroids, 1-year OS, and patient-reported outcomes. Crossover from the placebo group to open-label treatment with siltuximab was allowed after first treatment failure. 


\section{Results}

After a median duration of follow-up in the intent-to-treat population of 422 days, $34 \%$ patients in the siltuximab-treated group showed a durable response (1 CR, $17 \mathrm{PR}$ ) compared to 0 in the placebo group. Notably, no patients with the hyaline vascular subtype attained a durable response. As in preceding studies, baseline IL-6 levels did not correlate with response. Treatment with siltuximab resulted in a reliable increase in albumin as well as a decrease in CRP, although authors could not identify a baseline CRP level that would predict response. One-year OS was $100 \%$ in the siltuximab arm and $92 \%$ in the placebo arm; other secondary endpoints are summarized in Table 1.

Median time to radiologic response was 155 days, with median duration of response reported at 383 days. In the siltuximab-treated group, median duration of blinded treatment was 375 days, with 19 cycles of treatment, compared to 152 days ( 8 cycles) in the placebo group. Similarly, median time to treatment failure was not reached in the siltuximab group compared to 134 days for patients who received placebo. Around $40 \%$ of siltuximab-treated patients had $\geq 1$ dose delayed, with $3 \%$ total doses of siltuximab delayed due to AEs in 15 patients, most frequently neutropenia $(n=2)$. Due to treatment failure, $30 \%$ of patients in the siltuximab arm discontinued drug, while $50 \%$ (13) placebo-treated patients crossed over to receive open-label siltuximab. At time of analysis, one of these patients attained PR, and 9 others had not yet reached treatment failure.

\section{Safety}

Despite a significantly longer treatment time for the siltuximab group, AEs occurred at similar frequencies in both groups, with $23 \%$ incidence of severe AE (SAE) in siltuximab-treated patients compared to $19 \%$ in the placebo arm. AEs more likely to occur with siltuximab treatment included pruritus, rash, weight gain, upper-respiratory infection, and edema. Four siltuximab-treated patients experienced low-grade infusion reaction, with one case of anaphylaxis. AEs that led to drug discontinuation (23\% in the siltuximab group vs $38 \%$ in the placebo group) were all attributed to disease progression, except in the case of the anaphylactic infusion reaction and in a patient on placebo who developed myelodysplastic syndrome. Fatigue and night sweats were the only grade $\geq 3$ events that occurred in $>5 \%$ of siltuximab-treated patients. Three patients had siltuximab-related SAEs (sepsis, lower-respiratory infection, and anaphylaxis), but there were no siltuximab-related

Table I Key efficacy endpoints

\begin{tabular}{|c|c|c|c|c|}
\hline & $\begin{array}{l}\text { Siltuximab } \\
\text { group }(n=53)\end{array}$ & $\begin{array}{l}\text { Placebo } \\
\text { group }(n=26)\end{array}$ & $\begin{array}{l}\text { Difference or } \\
\text { HR }(95 \% \mathrm{CI})\end{array}$ & P-value \\
\hline \multicolumn{5}{|l|}{ Primary endpoint } \\
\hline Durable tumor and symptomatic response by independent review* & $18(34 \%)$ & $0(0 \%)$ & $34.0 \%(11.1-54.8)$ & 0.0012 \\
\hline Complete response & I (2)\% & $0(0 \%)$ & - & - \\
\hline Partial response & $17(32 \%)$ & $0(0 \%)$ & - & - \\
\hline Duration of durable tumour and symptomatic response* (days) & $383(232-676)$ & - & - & - \\
\hline \multicolumn{5}{|l|}{ Secondary endpoints } \\
\hline Tumour response by independent review ${ }^{\dagger}$ & $20(38 \%)$ & I (4\%) & $33.9 \%(11.1-54.8)$ & 0.0022 \\
\hline Complete response & $2(4 \%)$ & $0(0 \%)$ & - & - \\
\hline Partial response & $18(34 \%)$ & $\mathrm{I}(4 \%)$ & - & - \\
\hline Tumour response by investigator Assessment ${ }^{\dagger}$ & $27(51 \%)$ & $0(0 \%)$ & $50.9 \%(29.2-70.1)$ & $<0.0001$ \\
\hline Complete response & $3(6 \%)$ & $0(0 \%)$ & - & - \\
\hline Partial response & $24(45 \%)$ & $0(0 \%)$ & - & - \\
\hline Time to tumour response by independent review for responders ${ }^{\dagger}$ (days) & $155(44-742)$ & $65(65-65)$ & - & - \\
\hline Durable symptomatic response rate* & $30(57 \%)$ & $5(19 \%)$ & $37.4 \%(14.9-58.2)$ & 0.0018 \\
\hline Complete symptom response & $13(25 \%)$ & $0(0 \%)$ & $24.5 \%(1.4-46.2)$ & 0.0037 \\
\hline Time to durable symptomatic response* (days) & $170(67-274)$ & NE (227-NE) & $2.774(1.068-7.206)$ & 0.0288 \\
\hline Time to treatment failure* (days) & NE (378-NE) & I 34 (85-NE) & $0.418(0.214-0.815)$ & 0.0084 \\
\hline Time to next treatment* (days) & NE (NE-NE) & 280 (I6I-NE) & $0.298(0.137-0.652)$ & 0.0013 \\
\hline $\begin{array}{l}\text { Haemoglobin concentration increase of } \geq 15 \mathrm{~g} / \mathrm{L} \text { at week } 13 \\
\text { compared with baseline } \mathrm{f}^{\ddagger}\end{array}$ & $19(61 \%)$ & $0(0 \%)$ & $61.3(28.3-85.1)$ & 0.0002 \\
\hline Patients who discontinued corticosteroids $\$$ & $4(3 \mid \%)$ & I (II\%) & $19.7(-23.6-56.7)$ & 0.3602 \\
\hline
\end{tabular}

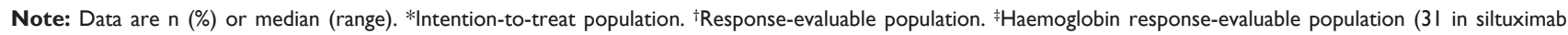
group vs II in placebo group). \$Patients taking corticosteroids at baseline: 13 vs nine. Reprinted from Lancet Oncol, Vol I5, van Rhee F, Wong RS, Munshi N, et al, Siltuximab for multicentric Castleman's disease: a randomised, double-blind, placebo-controlled trial, pages 966-974, Copyright 2014, with permission from Elsevier. ${ }^{23}$ Abbreviations: $\mathrm{NE}$, not evaluable; $\mathrm{Cl}$, confidence interval; $\mathrm{HR}$, hazard ratio. 
Table 2 Most common adverse events of all grades by siltuximab trial

\begin{tabular}{ll}
\hline $\begin{array}{l}\text { Adverse events in clinical } \\
\text { studies }\end{array}$ & $\begin{array}{l}\text { \% of adverse } \\
\text { events }\end{array}$ \\
\hline Phase I (Kurzrock et al) ${ }^{24}$ & 25 \\
Thrombocytopenia & 19 \\
Neutropenia & 19 \\
Hypertriglyceridemia & 18 \\
Leukopenia & 15 \\
Hypercholesterolemia & \\
Phase II (van Rhee et al) & \\
URI & 89 \\
Nausea & 63 \\
Vomiting & 58 \\
Diarrhea & 53 \\
Hypercholesterolemia & 15 \\
RCT (van Rhee et al) & \\
Pruritus & \\
URI & 42 \\
Fatigue & 36 \\
Rash & 34 \\
Peripheral edema & 34 \\
\hline Ab & 32 \\
\hline
\end{tabular}

Abbreviations: RCT, randomized, controlled trial; URI, upper respiratory tract infection.

deaths. Table 2 summarizes AEs reported in all three siltuximab trials.

\section{Discussion}

Evaluating treatment efficacy in CD presents several challenges, given its status as a rare, heterogeneous disease. First, there is no standard for classifying CD, although a recent paper in Blood suggests categorization based on HHV-8 association and posits the term "idiopathic multicentric CD" (iMCD) as a subclassification that largely describes the population studied in the above trials; that is, patients with multicentric disease without HHV-8 or HIV association. ${ }^{4}$ It remains unclear what percentage of CD patients comprise this subclassification, and how their prognosis and response to treatment compare to those with HHV-8-associated disease; of note, a recent systematic literature review published as an abstract identified $32 \%$ of HIV-negative cases as HHV-8-negative and 26\% as HHV-8 unknown. ${ }^{28}$ Further adding to the uncertainty of classification is the fact that there is no standard method for establishing HHV-8 positivity: some experts believe seropositivity alone to be an unreliable test, instead recommending detection of replicating virus by quantitative polymerase chain reaction in the blood or positive LANA-1 stain of the lymph node. Broad adoption of the subclassification system recommended by Fajgenbaum et $\mathrm{al}^{4}$ could help to clear much of the ambiguity surrounding the diagnosis of CD and set the stage for better standardization in further studies assessing prognosis and response to treatment.

Second, there is no standard of care in CD. Studies of other therapies are limited to case series and retrospective reviews, with the exception of the 2005 Japanese study evaluating tocilizumab, the monoclonal antibody to IL-6-receptor. Although that Phase II trial led to approval of tocilizumab for treatment of multicentric CD in Japan, it lacked a control group and utilized less rigorous criteria for response. Additionally, the 28 patients enrolled represented a much more homogenous group than those studied in the siltuximab trials.

Third, there is no current standard for assessing disease response in CD. Although both of the above siltuximab studies adopted some form of the Cheson criteria to define radiologic response, their approaches to defining symptomatic response were slightly different. The Phase I study defined CBR in terms of improvement in anemia, fatigue, anorexia, fever, weight, and/or lymph node diameter, evaluated on days 37 and 56 and "during extended treatment". The randomized Phase II trial, on the other hand, assessed symptomatic response based on improvement or stabilization of the sum of the severity of 34 CD-related signs and symptoms after 18 weeks of treatment or more. Again, standardization of response assessment will be helpful to evaluate the comparative efficacy of treatment strategies in CD.

Even in light of these inherent limitations, siltuximab appears to be a safe treatment option for treatment of HIV-negative, HHV-8-negative multicentric CD as evidenced by the safety profiles in all three studies. Moreover, while there is no standard of care to which it can be compared, siltuximab seems at least to outperform placebo in the first-ever randomized controlled trial in CD in terms of durable tumor response as well as improvement in symptoms and laboratory values. Importantly, durable responses were seen in both newly diagnosed and previously treated patients. Although no statistically significant difference in OS between siltuximab and best supportive care was observed, the randomized study's cross-over design may have muted any potential survival advantage. In addition, as authors of this study point out, achievement of radiologic response took much longer than correction of laboratory values. Since a proportion of patients in the siltuximab arm were still on treatment at the time of analysis (21\%), it is possible that some of these may attain response with continued treatment. A longer follow-up period could thus reveal additional responses or even uncover a survival advantage. 
It remains unclear why some patients achieved durable responses, while others did not. Subgroup analysis in the randomized Phase II trial showed increased benefit of siltuximab for patients younger than 65 , race other than white, male sex, and lack of corticosteroid use at randomization, which may serve as a proxy for disease severity. In that trial, no patients with hyaline vascular subtype attained response, but the previous Phase I study reported responses in this group. In both studies, baseline CRP and IL-6 levels do not appear to correspond with response. In addition, siltuximab's direct interaction with IL-6 limits the ability to assay IL-6 levels during treatment. It is possible that other cytokines (eg, IL-1, IL-10, TNF- $\alpha$ ) may drive disease in nonresponders, but further studies will be needed to address this question.

Although the above siltuximab trials provide valuable insight into treatment options for patients with "idiopathic" multicentric $\mathrm{CD}$, they do not address the efficacy of this drug in HHV-8-driven disease. The authors of the randomized trial report that siltuximab was not studied in this population since (per unpublished data) siltuximab does not bind to viral IL-6, the cytokine postulated to fuel HHV-8-associated CD. However, recent work suggests that human IL-6 also contributes to pathology in HHV-8-positive CD. ${ }^{29}$ As a whole, treatment for HIV- and HHV-8-associated CD remains understudied. Future studies should investigate whether the durable responses seen with siltuximab in HHV-8-negative $\mathrm{CD}$ patients can be recapitulated in patients with $\mathrm{HHV}-8$ positivity.

In conclusion, the first-ever randomized, controlled trial in multicentric CD posits siltuximab as a promising treatment option for patients with new and previously treated disease. Furthermore, the study represents an important initial step toward standardization of treatment approach in this poorly understood disorder. The Phase I and II studies evaluating the efficacy of single-agent siltuximab treatment lay the groundwork for future investigation of combination therapy with siltuximab-containing regimens; it is possible that combining siltuximab with tocilizumab, other cytokine inhibitors, or "conventional" CD treatments may improve response rate. Overall, much more work is needed to determine the optimal treatment strategy in multicentric CD.

\section{Disclosure}

SL is a consultant for Millennium, Celgene, Novartis, BMS, Janssen, and Onyx. The authors report no other conflicts of interest in this work.

\section{References}

1. Munshi N, Mehra M, van de Velde H, Desai A, Potluri R, Vermeulen J. Use of a claims database to characterize and estimate the incidence rate for Castleman disease. Leuk Lymphoma. 2014;1-9.

2. Casper, C. The aetiology and management of Castleman disease at 50 years: translating pathophysiology to patient care. $\mathrm{Br}$ J Haematol. 2005;129:3-17.

3. Cronin DM, Warnke RA. Castleman disease: an update on classification and the spectrum of associated lesions. Adv Anat Pathol. 2009;16:236-246.

4. Fajgenbaum DC, van Rhee F, Nabel CS. HHV-8-negative, idiopathic multicentric Castleman disease: novel insights into biology, pathogenesis, and therapy. Blood. 2014;123(19):2924-2933.

5. Frizzera G, Peterson BA, Bayrd ED, Goldman A. A systemic lymphoproliferative disorder with morphologic features of Castleman's disease: clinical findings and clinicopathologic correlations in 15 patients. J Clin Oncol. 1985;3:1202-1216.

6. Tsukamoto Y, Hanada N, Nomura Y, et al. Rapidly progressive renal failure associated with angiofollicular lymph node hyperplasia. Am J Nephrol. 1991;11:430-436.

7. Inoue M, Ankou M, Hua J, Iwaki Y, Hagihara M, Ota Y. Complete resolution of TAFRO syndrome (thrombocytopenia, anasarca, fever, reticulin fibrosis and organomegaly) after immunosuppressive therapies using corticosteroids and cyclosporin A: a case report. J Clin Exp Hematop. 2013;53:95-99.

8. Takai K, Nikkuni K, Momoi A, Nagai K, Igarashi N, Saeki T. Thrombocytopenia with reticulin fibrosis accompanied by fever, anasarca and hepatosplenomegaly: a clinical report of five cases. J Clin Exp Hematop. 2013;53:63-68.

9. Gerard L, Bérezné A, Galicier L, et al. Prospective study of rituximab in chemotherapy-dependent human immunodeficiency virus associated multicentric Castleman's disease: ANRS 117 CastlemaB Trial. J Clin Oncol. 2007;25:3350-3356.

10. Bower M, Powles T, Williams S, et al. Brief communication: rituximab in HIV-associated multicentric Castleman disease. Ann Intern Med. 2007;147:836-839.

11. Bower M, Newsom-Davis T, Naresh K, et al. Clinical features and outcome in HIV-associated multicentric Castleman's disease. J Clin Oncol. 2011;29:2481-2486.

12. Gholam D, Vantelon JM, Al-Jijakli A, Bourhis JH. A case of multicentric Castleman's disease associated with advanced systemic amyloidosis treated with chemotherapy and anti-CD20 monoclonal antibody. Ann Hematol. 2003;82:766-768.

13. Ide M, Kawachi Y, Izumi Y, Kasagi K, Ogino T. Long-term remission in HIV-negative patients with multicentric Castleman's disease using rituximab. Eur J Haematol. 2006;76:119-123.

14. Ocio E, Sanchez-Guijo FM, Diez-Campelo M, et al. Efficacy of rituximab in an aggressive form of multicentric Castleman disease associated with immune phenomena. Am J Hematol. 2005;78:302-305.

15. Chronowski GM, Ha CS, Wilder RB, Cabanillas F, Manning J, Cox JD. Treatment of unicentric and multicentric Castleman disease and the role of radiotherapy. Cancer. 2001;92:670-676.

16. Herrada J, Cabanillas F, Rice L, Manning J, Pugh W. The clinical behavior of localized and multicentric Castleman disease. Ann Intern Med. 1998;128:657-662.

17. Talat N, Schulte KM. Castleman's disease: systematic analysis of 416 patients from the literature. Oncologist. 2011;16:1316-1324.

18. Dispenzieri A, Armitage JO, Loe MJ, et al. The clinical spectrum of Castleman's disease. Am J Hematol. 2012;87:997-1002.

19. Tanaka T, Kishimoto T. Targeting interleukin-6: all the way to treat autoimmune and inflammatory diseases. Int J Biol Sci. 2012; 8:1227-1236

20. Brandt SJ, Bodine DM, Dunbar CE, Nienhuis AW. Dysregulated interleukin 6 expression produces a syndrome resembling Castleman's disease in mice. J Clin Invest. 1990;86:592-599. 
21. Katsume A, Saito H, Yamada Y, et al. Anti-interleukin 6 (IL-6) receptor antibody suppresses Castleman's disease like symptoms emerged in IL-6 transgenic mice. Cytokine. 2002;20:304-311.

22. Nishimoto N, Kanakura Y, Aozasa K, et al. Humanized anti-interleukin-6 receptor antibody treatment of multicentric Castleman disease. Blood. 106:2627-2632.

23. van Rhee F, Wong RS, Munshi N, et al. Siltuximab for multicentric Castleman's disease: a randomised, double-blind, placebo-controlled trial. Lancet Oncol. 2014;15:966-974.

24. Kurzrock R, Voorhees PM, Casper C, et al. A phase I, open-label study of siltuximab, an anti-IL-6 monoclonal antibody, in patients with B-cell non-Hodgkin lymphoma, multiple myeloma, or Castleman disease. Clin Cancer Res. 2013;19:3659-3670.

25. Cheson BD, Pfistner B, Juweid ME, et al. Revised response criteria for malignant lymphoma. J Clin Oncol. 2007;25:579-586.
26. van Rhee F, Fayad L, Voorhees P, et al. Siltuximab, a novel anti-interleukin-6 monoclonal antibody, for Castleman's disease. J Clin Oncol. 2010;28:3701-3708.

27. van Rhee F, Casper C, Voorhees PM, et al. An open-label, phase 2, multicenter study of the safety of long-term treatment with siltuximab (an anti-interleukin-6 monoclonal antibody) in patients with multicentric Castleman's disease. Blood. 2013;122:1806.

28. Fajgenbaum DC, Liu A, Ruth J, et al. HHV-8-negative, idiopathic multicentric Castleman disease (iMCD): a description of clinical features and therapeutic options through a systematic literature review. Blood. 2014; $124: 4861$.

29. Polizzotto MN, Uldrick TS, Wang V, et al. Human and viral interleukin-6 and other cytokines in Kaposi sarcoma herpesvirus-associated multicentric Castleman disease. Blood. 2013;122:4189-4198.
Biologics: Targets \& Therapy

\section{Publish your work in this journal}

Biologics: Targets \& Therapy is an international, peer-reviewed journal focusing on the patho-physiological rationale for and clinical application of Biologic agents in the management of autoimmune diseases, cancers or other pathologies where a molecular target can be identified. This journal is indexed on PubMed Central, CAS, EMBase, Scopus

Submit your manuscript here: http://www.dovepress.com/biologics-targets--therapy-journal

\section{Dovepress}

and the Elsevier Bibliographic databases. The manuscript management system is completely online and includes a very quick and fair peerreview system, which is all easy to use. Visit http://www.dovepress. com/testimonials.php to read real quotes from published authors. 